\title{
DANO DE FRIO EM LIMAS-ÁCIDAS TAHITI, COLHIDAS EM DIFERENTES ÉPOCAS E SUBMETIDAS A TRATAMENTOS TÉRMICOS E BIOQUÍMICOS ${ }^{1}$
}

\author{
LEANDRO CAMARGO NEVES ${ }^{2}$, RONALDO MORENO BENEDETTE ${ }^{3}$, VANUZAXAVIER DA SILVA ${ }^{3}$, \\ ROGÉRIO LOPES VIEITES ${ }^{4}$, SÉRGIORUFFO ROBERTO
}

RESUMO-O estudo objetivou o estabelecimento de um método efetivo e satisfatório do controle do dano de frio em limas-ácidas. Os frutos colhidos no município de Boa Vista-RR, 140 e 150 dias após a floração, apresentaram valores médios de 7,9 e 8,2 ${ }^{\circ}$ Brix; 6,3 e 6,0 $\mathrm{mL}$ de ácido cítrico. $100 \mathrm{~mL}$ de polpa ${ }^{-1}$ e $\mathrm{pH}$ de 2,8 e 3,0, respectivamente, nas duas colheitas. Após cada colheita, os frutos foram levados ao laboratório de Fitotecnia/UFRR, onde foram selecionados, limpos e submetidos aos tratamentos: T1 - controle; T2, T3 e $\mathrm{T} 4$ - condicionamento a $35^{\circ} \mathrm{C}$, por 6,12 e 24 horas, respectivamente; $\mathrm{T} 5$-aquecimento intermitente a $20^{\circ} \mathrm{C}$, por 8 horas, após 5 e 10 dias a $1{ }^{\circ} \mathrm{C}$; $\mathrm{T} 6$ - aquecimento intermitente a $20^{\circ} \mathrm{C}$, por 8 horas, após 10 e 20 dias a $1{ }^{\circ} \mathrm{C} ; \mathrm{T} 7$ - ethephon a $1.500 \mathrm{mg} . \mathrm{L}^{-1} ; \mathrm{T} 8$ - ethephon a $3.000 \mathrm{mg} \cdot \mathrm{L}^{-1}$. Os tratamentos T9 ao T16, diferenciaram-se dos tratamentos T1 a T8, apenas, na data da colheita (10 dias após a primeira). O experimento foi avaliado a cada 15 dias, durante 75 dias, a $1 \pm 0,5^{\circ} \mathrm{C}$ e $92 \pm 5 \%$ de UR, quanto ao dano de frio, aspecto visual, perda de massa fresca, sólidos solúveis (SS), acidez titulável (AT), SS/AT (ratio - RT), clorofila total e ácido ascórbico. O atraso na colheita não proporcionou efeito significativo algum. Todos os tratamentos, à exceção do controle e do aquecimento intermitente aos 10 e 20 dias, foram eficientes no controle do dano de frio. No entanto, o tratamento químico e o condicionamento térmico aceleraram precocemente o metabolismo dos frutos, principalmente no que concerne à perda de massa fresca e ao aspecto visual. O maior teor de clorofila total e de ácido ascórbico, bem como o melhor aspecto visual, a não-incidência de podridões e a menor perda de massa fresca foram detectadas nos frutos submetidos ao aquecimento intermitente aos 5 e 10 dias. Os SS, AT e RT estavam dentro dos padrões de qualidade e não variaram estatisticamente entre os tratamentos.

Termos para indexação: pós-colheita, Citrus latifolia Tanaka, qualidade, conservação.

\section{COLD DAMAGE IN TAHITI ACID LIMES HARVESTED IN DIFFERENT PERIODS AND SUBMITTED TO TERMICHAL AND BIOCHEMICAL TREATMENTS}

\begin{abstract}
The aim of this study was to establish an effective and satisfactory method to control chilling injuries on Tahiti lime. Thus, the fruits that were harvested in Boa Vista, RR, 140 and 150 days after flowering, showed average values of 7.9 and $8.2^{\circ}$ Brix and 6.3 and $6.0 \mathrm{~mL}$ of citric acid $/ 100 \mathrm{~mL}$ of flesh and 2.8 and $3.0 \mathrm{pH}$, respectively in two harvests. After the harvest the fruits were taken to the laboratory of Plant Production/UFRR, selected, cleaned and submitted to the following treatments: T1 - control; T2, T3 and T4 maintained at $35^{\circ} \mathrm{C}$ for 6,12 and 24 hours, respectively; T5 - intermittent warming at $20^{\circ} \mathrm{C}$ for 8 hours, after 5 and 10 days at $1{ }^{\circ} \mathrm{C}$; T6 -

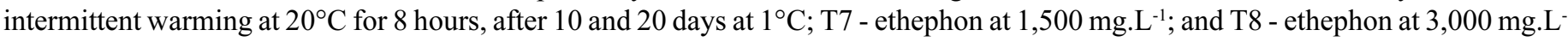
${ }^{1}$.The treatments $\mathrm{T} 9$ to $\mathrm{T} 16$, only differed from $\mathrm{T} 1$ to $\mathrm{T} 8$, only on the harvest date (10 days after the first one). The experiment was evaluated every 15 days, during 75 days at $1 \pm 0.5^{\circ} \mathrm{C}$ and $92 \pm 5 \%$ of RU, regarding the chilling injury, visual aspect, lack of fresh mass, soluble solids (SS), titratable acidity (TA), SS/TA (ratio - RT), total chlorophyll and ascorbic acid. The delay of the harvest did not provide any significative effect. All treatments, except the control and intermittent warming in 10 and 20 days, were efficient to control the chilling injury. However, the chemical and thermal conditioning speeded up the metabolism of fruits, mainly concerning the lack of fresh mass and visual aspect characteristics. The higher chlorophyll and ascorbic acid content, as well the best visual aspect, no rottenness incidence and the lower lack of fresh mass were detected on fruits submitted to the intermittent warming at 5 and 10 days. The SS, AT and RT were considered compatible to the quality standard and did not vary statistically among the treatments which had shown resistance to the chilling injury.
\end{abstract}

Index Terms: post harvest, Citrus latifolia Tanaka, quality, conservation.

1(Trabalho 130-07). Recebido em: 21-05-2007. Aceito para publicação em: 24-08-2007. Apoio: Roraima AgroFrutas.

${ }^{2}$ Prof.Depto de Fitotecnia da Universidade Federal de Roraima (UFRR), 69310-270, Boa Vista-RR. E-mail: rapelbtu@hotmail.com.

${ }^{3}$ Graduandos em Agronomia da Universidade Federal de Roraima (UFRR), 69310-270, Boa Vista-RR. E-mail:rmbenedette@hotmail.com; vanuzaxs@hotmail.com.

${ }^{4}$ Prof. do Depto de Gestão e Tecnologia Agroindustrial da Universidade Estadual de São Paulo/UNESP, 18603-970, Botucatu-SP. E-mail: :vieites@fcav.unesp.br.

${ }^{5}$ Prof,.do Depto. De Agronomia daUniversidade Estadual de Londrina (UEL), 86051-990, Londrina-PR. E-mail:sroberto@uel.br. 


\section{INTRODUÇÃO}

No Brasil, a lima-ácida Tahiti (Citrus latifolia Tanaka) destaca-se hoje como um dos frutos cítricos de maior importância comercial, estimando-se a produção em 1 milhão de toneladas/ ano, em aproximadamente 40 mil ha (Agrianual, 2004). O Estado de São Paulo é o maior produtor e exportador brasileiro, com área superior a $30 \mathrm{mil} \mathrm{ha,} \mathrm{sendo} \mathrm{responsável} \mathrm{por} \mathrm{quase} 70 \%$ da produção brasileira (EMBRAPA, 2007). Exemplificando, do volume comercializado no CEAGESP em 2003, ou seja, 66.017 ton. de limas-ácidas, foram exportadas 34.012 ton., ou seja, mais de $50 \%$ do total, o que representa rendimento na ordem de US\$ 16.500.000 (Agrianual, 2005).

No entanto, analisando containers com limas-ácidas Tahiti a serem exportadas por empresas baianas, para o mercado europeu, identificaram-se problemas que resultaram em perdas consideráveis, que, em determinados momentos, ultrapassaram a $20 \%$ do total (Coelho, 2007). Assim, torna-se cada vez mais necessário investir em tecnologias para manutenção da qualidade em pós-colheita, principalmente quando os frutos forem destinados à exportação.

São várias as razões para essas perdas, como a colheita precoce ou tardia frente ao ponto ideal e, em pós-colheita, podemse destacar os danos causados pela exposição prolongada das limas-ácidas a temperaturas abaixo de $10^{\circ} \mathrm{C}$ (Kluge et al., 2003a/ b; Jomori et al., 2003a/b). Esses danos, também chamados de fisiopatias, inicialmente desenvolvidos durante o frigoarmazenamento, e manifestados após a saída dos frutos da câmara frigorífica, são caracterizados por depressões superficiais na casca e aumento na incidência de doenças, além da perda excessiva de umidade e a manifestação de flavors nãocaracterísticos.

Segundo Chitarra \& Chitarra (2005), a temperatura recomendada para o armazenamento refrigerado seguro da limaácida Tahiti é de 9 a $10^{\circ} \mathrm{C}$. Abaixo, é quase que inevitável o surgimento desses distúrbios fisiológicos. Com isso, é possível pressupor que frutos sensíveis a baixas temperaturas, podem ter a vida útil comprometida, visto que não podem ser frigoarmazenados em temperaturas que satisfatoriamente desaceleraram o metabolismo desses frutos. Entretanto, como citado por Kluge et al. (2006), mesmo não existindo método integralmente efetivo no controle dos sintomas desencadeados pela constante exposição dos frutos a baixas temperaturas, alguns tratamentos térmicos e químicos têm-se mostrado satisfatórios. Essas técnicas consistem na redução e/ou no retardamento do desenvolvimento dessas sintomatologias ou pelo aumento da tolerância às baixas temperaturas durante o frigoarmazenamento dos frutos (Jomori et al., 2005). Dentre essas técnicas, destacamse os tratamentos térmicos aplicados antes da refrigeração, bem como o condicionamento térmico ou durante o período de frigoarmazenamento, na forma de aquecimento intermitente.

Assim, o condicionamento térmico tem reduzido os danos causados pelas baixas temperaturas em limas-ácidas Tahiti (Jomori, 2005), além de diminuir, também, a incidência de podridões em laranjas e pomelos (Porat et al., 1999; Rodov et al.,
2000). Nesse sentido, embora a elevação da temperatura possa causar a perda excessiva de umidade e de firmeza de polpa, o tratamento térmico também pode ser utilizado para a desinfecção microbiana. Dessa forma, se bem estabelecido o binômio tempo $\mathrm{x}$ temperatura, esse tratamento térmico pode aumentar a termotolerência dos frutos sem, contudo, acelerar a sua senescência. Da mesma maneira, a eficiência no controle dos danos pelo frio, com a aplicação do aquecimento intermitente, também foi verificada em pomelos e laranjas (Schirra \& Cohen, 1999), e em limas-ácidas Tahiti (Kluge et al., 2006). No entanto, o envolvimento do etileno, no controle das desordens fisiológicas durante o frigoarmazenamento de limas-ácidas Tahiti, ainda não ficou bem esclarecido. Jomori (2005) relata que a adição ou supressão desse regulador em limas-ácidas Tahiti não demonstrou diferença quanto ao controle dos danos ocasionados pelo frio, quando em comparação aos frutos-controle. Já em abacates (Lee \& Yong, 1984), limas-ácidas Tahiti e algumas variedades cítricas (Yuen et al., 1995), verificaram-se aumentos na incidência desses danos pelo frio nos frutos submetidos à aplicação do etileno exógeno. Entretanto, a aplicação do 1-MCP em abacaxis (Selvarajah et al., 2001), em abacates (Pesis et al., 2002) e em caquis (Salvador et al., 2004) mostrou-se efetivo na contenção dos danos de frio.

Com isso, diante da problemática a respeito dos danos causados pelo frio em limas-ácidas Tahiti e da não-consolidação de um controle conclusivamente eficiente, o presente trabalho tem por objetivo determinar um método eficiente no controle e/ ou na diminuição desses danos, durante o frigoarmazenamento de limas-ácidas Tahiti.

\section{MATERIAL E MÉTODOS}

Os frutos foram colhidos em propriedade agrícola situada em Boa Vista-RR, localizada na latitude $2^{\circ} 50^{\prime} 06^{\prime \prime} \mathrm{N}$ e longitude $60^{\circ} 40^{\prime} 28^{\prime \prime}$ W. Na colheita aos 140 dias, as limas-ácidas Tahiti apresentavam coloração verde-brilhante, massa média de $87 \pm$ 2,5g, SS médios de $7,7^{\circ}$ Brix, AT média de 6,3 de ácido cítrico. $100 \mathrm{~mL}_{\text {de }}$ polpa $^{-1}$ e pH médio de 2,8. Na colheita aos 150 dias, as limas-ácidas Tahiti apresentavam coloração verdebrilhante, massa média de $92 \pm 2 \mathrm{~g}$, SS médios de $7,9^{\circ} \mathrm{Brix}$, AT média de 6,0 de ácido cítrico. $100 \mathrm{~mL}$ de polpa ${ }^{-1}$ e $\mathrm{pH}$ médio de 3,0 . Após as colheitas, os frutos foram levados até o Laboratório de Fitotecnia $\left(20 \pm 2^{\circ} \mathrm{C}\right.$ e $75 \pm 3 \%$ de U.R.) da UFRR, sendo então selecionados pela ausência de defeitos visuais e higienizados em solução de hipoclorito de sódio $(\mathrm{NaOCl})$ a $100 \mathrm{mg} . \mathrm{L}^{-1}$, previamente acidificada, por 10 minutos. Realizado o enxágüe e a secagem em bandejas plásticas perfuradas expostas ao ar atmosférico, os frutos foram resfriados em B.O.D. por 10 horas, a $10 \pm 0,5^{\circ} \mathrm{C}$.

Para a constituição dos tratamentos, as limas-ácidas Tahiti, já acondicionadas em bandejas plásticas (30 frutos/ bandeja, correspondendo a $3 \pm 0,060 \mathrm{~kg}$ ), nas duas épocas de colheita, foram termicamente condicionadas em câmara do tipo B.O.D., regulada para a temperatura de $35 \pm 1^{\circ} \mathrm{C}$ e $90 \pm 3 \%$ de U.R. (mantida através de umidificadores), por 6; 12 e 24 horas, antes 
do armazenamento dos frutos a $1{ }^{\circ} \mathrm{C}$ e $93 \pm 2 \%$ de U.R.. Para os tratamentos submetidos ao aquecimento intermitente, procedimento semelhante foi realizado. Entretanto, primeiramente, os frutos foram armazenados nas condições já citadas, sendo posteriormente condicionados a $20 \pm 1{ }^{\circ} \mathrm{C}$ e $90 \pm 3 \%$ de U.R., por 8 horas, após 5 e 10 , e 10 e 20 dias de armazenamento refrigerado. Nos tratamentos submetidos ao acelerador de maturação, os frutos foram imersos em soluções contendo 1.500 e 3.000 mg.L $\mathrm{L}^{-1}$ de ethephon, por 10 minutos, sendo colocados para secar em condição ambiente. Dessa forma, os tratamentos ficaram assim dispostos:

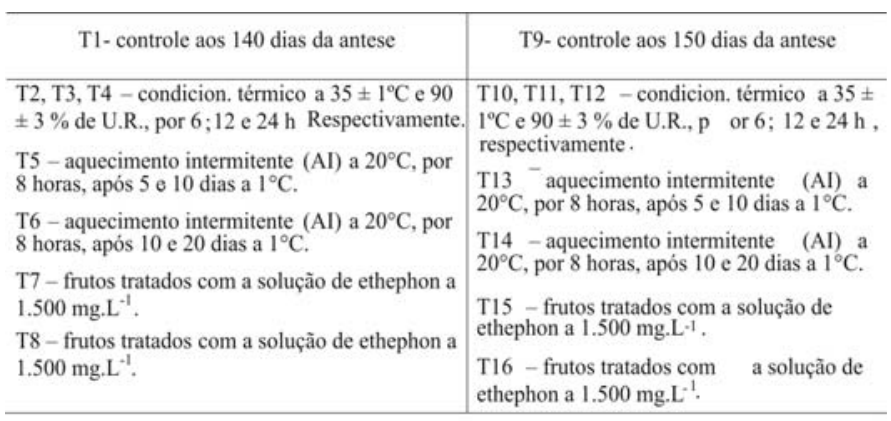

O experimento foi avaliado a cada 15 dias (6 horas após a saída dos frutos da câmara frigorífica), durante 75 dias de armazenamento a $1 \pm 0,5^{\circ} \mathrm{Ce} 92 \pm 5 \%$ de UR, quanto às seguintes variáveis:

1 - Danos causados pelo frio: foram determinados pela porcentagem de frutos injuriados (depressões na casca), estimando-se a severidade dessas injúrias de acordo com metodologia adaptada de Sala \& Lafuente (1999), sendo assim:0 $=$ nenhuma depressão superficial $(0 \%$ da superfície afetada $) ; 1=$ pouco danificado ( 1 a $5 \%$ da superfície afetada); $2=$ dano moderado (5 a $25 \%)$; 3 = dano severo $(25$ a $50 \%$ ), e $4=$ dano muito severo ( $>50 \%$ da superfície afetada).

2 - Aspecto visual dos frutos: foi quantificado, envolvendo conjuntamente duas variáveis, a perda de rugosidade e o murchamento. Quanto à perda de rugosidade dos frutos, estabeleceu-se um padrão inicial e, a partir daí, quinzenalmente, dez avaliadores atribuíram valores em uma escala nãosegmentada, do tipo 0 a 9 , onde 9 correspondeu a totalmente rugoso e 0 correspondeu a totalmente liso. Quanto ao murchamento dos frutos, o mesmo procedimento foi adotado, onde 9 correspondeu a totalmente túrgido e 0 a totalmente flácido e seco. Para a formulação do resultado da análise do aspecto visual, considerou-se a média aritmética das três características mencionadas acima (rugosidade, murchamento e podridões), para cada dia de análise.

3 - Presença de podridões: os frutos foram submetidos a análises microbiológicas para a determinação somente do gênero do agente causal (Vanderzant \& Splittstoesser, 1992), sendo posteriormente visualizadas através de fotografias de colônias.

4 - Perda de massa fresca dos frutos: foi expressa em porcentagens médias obtidas nas bandejas plásticas, considerando-se apenas a relação entre a massa fresca inicial de cada bandeja e a massa fresca obtida após cada análise. Para essa análise, 10 amostras por repetição foram usadas somente para esse propósito, pelo fato de a análise não ser destrutiva. 5 - Teor de sólidos solúveis (SS) dos frutos: foi determinado pela leitura refratométrica direta, com o refratômetro tipo Abbe, marca ATAGO - N1, e os resultados expressos em ${ }^{\circ}$ Brix.

6 - Nível de acidez titulável (AT) dos frutos: foi determinado por titulometria de neutralização, por titulação de $10 \mathrm{~g}$ de polpa, homogeneizada e diluída para $90 \mathrm{~mL}$ em água ultrapura, com solução padronizada de hidróxido de sódio a 1,0 $\mathrm{N}$, com ponto de viragem no $\mathrm{pH} 8,2$. Os resultados foram expressos em $\mathrm{mL}$ de ácido cítrico. $100 \mathrm{~g}$ de polpa ${ }^{-1}$.

7-Ratio: foi calculado pela divisão do teor de SS pela AT.

8 - Quantidade de clorofila total: foi determinada segundo metodologia de Manfroi et al. (1996). Assim, de cada amostra, foram retirados $10 \mathrm{~cm}^{2}$ da casca da região equatorial do fruto, na forma de uma tira de $1 \mathrm{~cm}$ de largura e $10 \mathrm{~cm}$ de comprimento. As tiras foram, então, colocadas em frascos de vidro herméticos $(220 \mathrm{~mL})$, contendo $20 \mathrm{~mL}$ de solução extratora de acetona a 80 $\%$. Os frascos foram revestidos com papel alumínio e mantidos a $4 \pm 1{ }^{\circ} \mathrm{C}$, por $72 \mathrm{~h}$, quando se procedeu à leitura da absorbância da solução em um espectrofotômetro Shimadzu Modelo UV-3600UV-VIS-NIR, nos comprimentos de onda de 645 e $663 \mathrm{~nm}$. O teor de clorofila total foi obtido pela seguinte fórmula: Clorofila total: 8,0 (Absorbância a $663 \mathrm{~nm})+20,2$ (Absorbância a $645 \mathrm{~nm})$. Os resultado foram expressos em $\mathrm{mg} .\left(40 \mathrm{~cm}^{2}\right)^{-1}$.

9 - Teor de ácido ascórbico: foi determinado segundo método de Carvalho et al. (1990), que se baseia na redução do indicador 2,6 - diclorofenol indolfenol (DCFI) pelo ácido ascórbico. A técnica baseia-se no preparo de uma alíquota de 10 $\mathrm{mL}$ de suco colocada em erlenmeyer contendo $50 \mathrm{~mL}$ de solução de ácido oxálico a $1 \%$. A titulação foi então efetuada com DCFI até atingir a coloração rosada persistente por 15 segundos. Os resultados foram expressos em $\mathrm{mL}$ de ácido ascórbico. $100 \mathrm{~mL}$ de polpa $^{-1}$.

Realizada uma análise exploratória dos dados, constatouse que os mesmos seguem uma distribuição normal, os erros são independentes e apresentam homocedasticidade. Assim, os dados foram submetidos à análise de variância, pelo teste $\mathrm{F}$, e a comparação de médias foi efetuada pelo teste de Tukey DMS, a $5 \%$ de probabilidade. O delineamento experimental empregado foi o inteiramente casualizado, seguindo um esquema fatorial 2x6x3 (duas épocas de colheita, seis tratamentos térmicos e três doses de ethephon) com três repetições e 10 amostras por repetição.

\section{RESULTADOS E DISCUSSÃO}

Os teores de SS, os níveis de AT e os valores da relação entre ambos não apresentaram diferenciação estatística entre os tratamentos testados. No entanto, vale ressaltar que todos os valores obtidos nessas análises estavam dentro dos padrões considerados adequados à comercialização de limasácidas Tahiti, semelhantes, por sua vez, aos valores apresentados nos estudos de Jomori et al. (2003a/b), Kluge et al. (2003a/b) e Biase \& Zanette (2000). As alterações na composição química, à exceção dos teores de ácido ascórbico e da quantidade de clorofila 
total, detectadas nos frutos, indicaram que a ação do ethephon e dos tratamentos térmicos ficou restrita mais ao albedo e ao flavedo dos frutos, não havendo, portanto, a atuação mais internalizada desses tratamentos. De fato, o fruto cítrico, além dessas barreiras iniciais, apresenta a sua estrutura interna segmentada através de membranas semipermeáveis, dificultado, assim, a entrada e a saída de substâncias (Jomori et al., 2003b). No entanto, os frutos submetidos ao aquecimento intermitente, aos 5 e 10 dias, apresentaram os maiores teores de ácido ascórbico e de clorofila total (Tabela 2 e 3), bem como as menores perdas de massa fresca (Tabela 4) e o melhor aspecto visual (Figura 2).

A exemplo das análises de SS, AT e ratio, os teores de ácido ascórbico também apresentaram constantes decréscimos (Tabela 2). Contudo, isso, até certo ponto, era previsto, pois o teor de ácido ascórbico em limas-ácidas Tahiti, bem como na maioria dos frutos, pode diminuir durante a pós-colheita. $\mathrm{O}$ diferencial então consistiria em quanto cada fruto conseguiria mantê-lo, mesmo após os tratamentos térmicos e químicos que supostamente acelerariam a maturação das limas-ácidas Tahiti. Nesse sentido, os frutos submetidos ao aquecimento intermitente aos 5 e 10 dias de armazenamento refrigerado, mais uma vez, apresentaram os melhores resultados, denotando-se perdas de não mais do que $29 \pm 1 \%$ dos teores iniciais de ácido ascórbico nos frutos. Esses resultados são considerados importantes, pois o ácido ascórbico, além das suas propriedades nutracêuticas já conhecidas, pode também atuar como agente antioxidante, contribuindo positivamente na preservação da qualidade durante o armazenamento refrigerado. Já, nos demais tratamentos, os frutos apresentaram perdas médias de $84 \pm 1,5 \%$ (controle) a 52 $\pm 3 \%$ (condicionamento térmico por 6 horas e ethephon na concentração de $1.500 \mathrm{~mL} \cdot \mathrm{L}^{-1}$ ). Essas perdas podem ter ocorrido pelo fato de que os ácidos orgânicos, dentre os quais o ascórbico, são consumidos em reações oxidativas durante a maturação e a senescência dos frutos. Jomori (2005) também descreveu em seus estudos que o aquecimento intermitente foi o tratamento que menos afetou os teores de ácido ascórbico em limas-ácidas Tahiti. No entanto, as perdas observadas foram na ordem de até $20 \%$, ou seja, menor que o melhor resultado encontrado no presente estudo. Situação semelhante foi observada por Schirra et al. (2005) em trabalho utilizando tratamentos térmicos, em laranjas sanguíneas. Contudo, Jomori (2005) conduziu seu experimento por apenas 45 dias, nas mesmas condições, enquanto aqui o experimento foi conduzido por 75 dias, apresentando apenas $9 \pm$ $1 \%$ a mais nas perdas dos teores de ácido ascórbico, contudo ganhando mais 25 dias de frigoconservação, o que comercialmente é muito interessante.

Quanto à análise da quantidade de clorofila total, os frutos submetidos ao aquecimento intermitente, aos 5 e 10 dias de armazenamento refrigerado, também se apresentaram superiores aos demais tratamentos. Ao final do período experimental, todos os frutos desse tratamento ainda apresentavam coloração predominantemente verde. Assim, atribuiu-se a esses frutos um estádio menos avançado de maturação em relação aos demais. Dessa forma, essas limas-ácidas Tahiti que ainda se apresentavam verdes ao final dos 75 dias de armazenamento refrigerado, além de estarem de acordo com os padrões de comercialização (Manfroi et al., 1996), também apresentavam maior potencial de conservação pós-colheita. Em trabalho similar, Jomori et al. (2003) também constataram o efeito do etileno, no entanto, através da sua supressão, com a exposição das limas-ácidas Tahiti ao 1$\mathrm{MCP}$, no que concerne à manutenção da coloração verde em limas-ácidas Tahiti. Goldschmidt (1997) também relatou que a degradação da clorofila é um evento metabólico que pode estar sendo regulado pela ação do etileno. Exemplificando então a ação do etileno no processo de desverdecimento em frutos cítricos, Jacomino et al. (2003), trabalhando com limões Siciliano, conseguiram desverdecer os frutos com a aplicação de $6 \mu \mathrm{L} . \mathrm{L}^{-1}$ de Azetil, que continha apenas $5 \%$ de etileno. Dessa forma, pelo estabelecimento de um controle entre o tempo e a temperatura do tratamento térmico, além da inibição dos danos de frio, o aquecimento intermitente aos 5 e 10 dias de armazenamento refrigerado proporcionou a melhor manutenção da clorofila total durante o período de todas as avaliações, potencializando, conseqüentemente, a comercialização futura desses frutos.

Em se tratando do dano de frio (Figura 1), o aquecimento intermitente aos 5 e 10 dias, o condicionamento térmico, em todos os tempos testados, bem como o tratamento com o ethephon, em ambas as concentrações, mostraram-se eficientes no controle dessas fisiopatias. Entretanto, à exceção do aquecimento intermitente aos 5 e 10 dias, os demais tratamentos, apesar do controle adequado dos danos fisiológicos, apresentaram perdas excessivas de massa fresca além do comprometimento visual dos frutos, presumindo-se, para esses tratamentos, qualidade inferior e substancialmente menor potencial de conservação. Esse comportamento pode ser devido à maior intensidade das reações oxidativas, mesmo havendo a contenção dos danos pelo frio, ocasionando a não-preservação da qualidade e da integridade física desses frutos. Da mesma forma, os frutos submetidos ao aquecimento intermitente aos 10 e 20 dias, justamente pelo atraso na aplicação do calor, resultaram numa tentativa frustrada no controle do dano de frio, além de, conseqüentemente, acelerar o metabolismo desses frutos. A aceleração metabólica, por sua vez, bem como a aplicação exógena de etileno ainda não foram contextualmente esclarecidas quanto à contenção dos danos de frio em frutos cítricos. Nesse sentido, Jomori (2005) menciona que, mesmo a aplicação exógena tendo demonstrado aumento da incidência do dano de frio em seu trabalho, outros autores mencionam que o mínimo deste regulador é extremamente necessário para o controle desses distúrbios fisiológicos, pois a presença mínima do etileno pode manter a atividade constante das enzimas antioxidativas. Contudo, no presente trabalho, o etileno exógeno, bem como os tratamentos térmicos, que possivelmente também tenham desencadeado a produção endógena de etileno (não quantificado), foram eficientes no controle dos danos de frio na limas-ácidas Tahiti.

Quanto à perda de massa fresca (Tabela 4), os frutos submetidos ao aquecimento intermitente, aos 5 e 10 dias, apresentaram perdas médias não-superiores a 5,32 \% em relação a sua massa inicial. Ao passo que os demais tratamentos apresentaram perdas médias variando de $12 ; 34$ a 20,11\%. Isso, seguramente, pode desqualificá-los perante o mercado consumidor, que preferem frutos completamente verdes, rugosos 
e túrgidos.

No aspecto visual dos frutos (Figura 2), que engloba duas das principais variáveis qualitativos dessa espécie, a rugosidade da epiderme e o nível de murchamento e/ou turgidez também puderam constatar que os frutos tratados termicamente pelo aquecimento intermitente, aos 5 e 10 dias, apresentaram os melhores índices. Nos outros tratamentos, em especial os frutos tratados termicamente por mais de 6 horas, e aqueles submetidos ao ethephon na concentração de $3.000 \mathrm{mg} . \mathrm{L}^{-1}$, apresentaram, elevada perda de massa fresca, o que, por sua vez, denota a esses frutos a característica de murchos e flácidos, com pouca ou quase nenhuma rugosidade. Nesse sentido, esses frutos passariam a impressão ao consumidor de velhos e passados, sendo, portanto, preteridos. Além disso, somente para o entendimento e caracterização da sintomatologia apresentada nos frutos contaminados, apenas nos frutos- controle e nos tratamentos submetidos ao ethephon, em ambas as concentrações, detectou-se a presença de Penicillium digitatum. Nos demais tratamentos, não foi detectada a presença de agentes microbianos. Tais circunstâncias, segundo os próprios autores, podem ser advindas da rápida aceleração metabólica nestes frutos e, supostamente, a potencialização de focos de contaminação já preexistentes. Nesse sentido, os autores mencionam que os tratamentos térmicos tenham sido suficientes para, além do controle dos danos de frio, a desinfecção satisfatória frente a possíveis contaminantes nos demais tratamentos. Para tanto, Jomori (2005) menciona que os tratamentos térmicos, embora possam, eventualmente, promover algum estresse oxidativo, podem, sobretudo, ser utilizados como tratamentos desinfetantes em frutos.

TABELA 1- Sólidos solúveis (SS), expresso em ${ }^{\circ} B r i x ;$ acidez titulável (AT, expressa em $\mathrm{mL}$ de ácido cítrico. $100 \mathrm{~mL}$ de polpa ${ }^{-1}$; e ratio, expresso através da relação SS/AT, em limas-ácidas Tahiti produzidas no Estado de Roraima, após 75 dias de frigoarmazenamento a $1 \pm 0,5^{\circ} \mathrm{C}$ e $92 \pm 3 \%$ de U.R., Boa Vista-RR -2006 .

\begin{tabular}{|c|c|c|c|}
\hline Tratamentos & Sólidos solúveis $\left({ }^{\circ}\right.$ Brix $)$ & $\begin{array}{l}\text { Acidez titulável ( } \mathrm{mL} \text { de ácido } \\
\left.\text { cítrico. } 100 \mathrm{~mL} \mathrm{de} \mathrm{polpa}{ }^{-1}\right)\end{array}$ & Ratio \\
\hline Controle -140 dias & $7,3 \mathrm{Aa}$ & $5,53 \mathrm{Aa}$ & $1,320 \mathrm{Aa}$ \\
\hline Cond. térmico 6 horas & 7,3 Aa & $5,54 \mathrm{Aa}$ & $1,318 \mathrm{Aa}$ \\
\hline Cond. térmico 12 horas & 7,3 Aa & $5,56 \mathrm{Aa}$ & $1,313 \mathrm{Aa}$ \\
\hline Cond. térmico 24 horas & $7,2 \mathrm{Aa}$ & $5,55 \mathrm{Aa}$ & $1,297 \mathrm{Aa}$ \\
\hline Aquecimento interm. 5/10 dias & 7,5 Aa & 5,59 Aa & $1,342 \mathrm{Aa}$ \\
\hline Aquecimento interm. 10/20 dias & $7,2 \mathrm{Aa}$ & $5,53 \mathrm{Aa}$ & $1,302 \mathrm{Aa}$ \\
\hline Ethephon 1.500 mg.L ${ }^{-1}$ & 7,3 Aa & $5,54 \mathrm{Aa}$ & $1,318 \mathrm{Aa}$ \\
\hline Ethephon 3.000 mg.L $^{-1}$ & $7,2 \mathrm{Aa}$ & $5,53 \mathrm{Aa}$ & $1,302 \mathrm{Aa}$ \\
\hline Controle -150 dias & $7,5 \mathrm{Aa}$ & $5,16 \mathrm{Aa}$ & $1,453 \mathrm{Aa}$ \\
\hline Cond. térmico 6 horas & 7,6 Aa & $5,20 \mathrm{Aa}$ & $1,462 \mathrm{Aa}$ \\
\hline Cond. térmico 12 horas & 7,6 Aa & $5,20 \mathrm{Aa}$ & $1,462 \mathrm{Aa}$ \\
\hline Cond. térmico 24 horas & 7,6 Aa & $5,19 \mathrm{Aa}$ & $1,464 \mathrm{Aa}$ \\
\hline Aquecimento interm. 5/10 dias & 7,7 Aa & $5,22 \mathrm{Aa}$ & $1,475 \mathrm{Aa}$ \\
\hline Aquecimento interm. 10/20 dias & 7,5 Aa & $5,18 \mathrm{Aa}$ & $1,448 \mathrm{Aa}$ \\
\hline Ethephon 1.500 mg.L $^{-1}$ & 7,6 Aa & $5,16 \mathrm{Aa}$ & $1,473 \mathrm{Aa}$ \\
\hline Ethephon 3.000 mg.L $^{-1}$ & 7,6 Aa & $5,17 \mathrm{Aa}$ & $1,470 \mathrm{Aa}$ \\
\hline
\end{tabular}

As médias seguidas das mesmas letras minúscula (linha) e maiúscula (coluna) não diferem entre si, ao nível de 5\% de probabilidade, pelo teste de Tukey.

Para as tabelas a seguir, estabelecem-se os seguintes tratamentos: T1 - controle aos 140 dias da antese; T2, T3, T4 condicionamento térmico a $35 \pm 1^{\circ} \mathrm{C}$ e $90 \pm 3 \%$ de U.R., por 6,12 e $24 \mathrm{~h}$, respectivamente; $\mathbf{T 5}$-aquecimento intermitente (AI) a $20^{\circ} \mathrm{C}$, por 8 horas, após 5 e 10 dias a $1{ }^{\circ} \mathrm{C} ; \mathbf{T 6}$ - aquecimento intermitente (AI) a $20^{\circ} \mathrm{C}$, por 8 horas, após 10 e 20 dias a $1{ }^{\circ} \mathrm{C}$; $\mathbf{T} 7$ - frutos tratados com a solução de ethephon a $1.500 \mathrm{mg} . \mathrm{L}^{-1} ; \mathbf{T} 8$ - frutos tratados com a solução de ethephon a $1.500 \mathrm{mg} . \mathrm{L}^{-1} ; \mathbf{T} 9$ - controle 150 dias da antese; T10, T11, T12 - condicionamento térmico a $35 \pm 1^{\circ} \mathrm{C}$ e $90 \pm 3 \%$ de U.R., por 6,12 e 24 h, respectivamente; T13 - aquecimento intermitente (AI) a $20^{\circ} \mathrm{C}$, por 8 horas, após 5 e 10 dias a $1{ }^{\circ} \mathrm{C} ; \mathbf{T 1 4}$ - aquecimento intermitente (AI) a $20^{\circ} \mathrm{C}$, por 8 horas, após 10 e 20 dias a $1^{\circ} \mathrm{C} ; \mathbf{T} 15$ - frutos tratados com a solução de ethephon a $1.500 \mathrm{mg} . \mathrm{L}^{-1} ; \mathbf{T 1 6}$ - frutos tratados com a solução de ethephon a $1.500 \mathrm{mg} . \mathrm{L}^{-1}$. 
TABELA 2 - Teor de ácido ascórbico (mg de ácido ascórbico.100g de polpa ${ }^{-1}$ ), em limas-ácidas produzidas no Estado de Roraima, frigoarmazenadas por 75 dias a $1 \pm 0,5^{\circ} \mathrm{C}$ e $92 \pm 3 \%$ de U.R., Boa Vista-RR -2006.

\begin{tabular}{c|cccccccc}
\hline & $\mathbf{T 1}$ & $\mathbf{T 2}$ & $\mathbf{T 3}$ & $\mathbf{T 4}$ & $\mathbf{T 5}$ & $\mathbf{T 6}$ & T7 & T8 \\
\hline $\mathbf{1 5}$ & $32,45 \mathrm{Ac}$ & $34,70 \mathrm{Ab}$ & $34,25 \mathrm{Ab}$ & $34,80 \mathrm{Ab}$ & $36,80 \mathrm{Aa}$ & $31,30 \mathrm{Ac}$ & $35,02 \mathrm{Ab}$ & $34,94 \mathrm{Ab}$ \\
$\mathbf{3 0}$ & $26,54 \mathrm{Ac}$ & $31,14 \mathrm{Aa}$ & $29,18 \mathrm{Ab}$ & $28,14 \mathrm{Ab}$ & $33,65 \mathrm{Aa}$ & $23,49 \mathrm{Bd}$ & $31,88 \mathrm{Aa}$ & $29,19 \mathrm{Ab}$ \\
$\mathbf{4 5}$ & $17,66 \mathrm{Cc}$ & $26,94 \mathrm{Cb}$ & $22,20 \mathrm{Bb}$ & $20,93 \mathrm{Bc}$ & $31,49 \mathrm{Ba}$ & $15,30 \mathrm{Cd}$ & $26,22 \mathrm{Cb}$ & $21,40 \mathrm{Bc}$ \\
$\mathbf{6 0}$ & $10,12 \mathrm{Dd}$ & $19,14 \mathrm{Db}$ & $16,33 \mathrm{Cc}$ & $15,77 \mathrm{Cc}$ & $29,28 \mathrm{Ba}$ & $8,42 \mathrm{Dd}$ & $19,90 \mathrm{Db}$ & $16,69 \mathrm{Cc}$ \\
$\mathbf{7 5}$ & $5,10 \mathrm{Dd}$ & $15,23 \mathrm{Db}$ & $9,03 \mathrm{Dc}$ & $8,73 \mathrm{Dc}$ & $27,44 \mathrm{Ca}$ & $5,30 \mathrm{Dd}$ & $15,33 \mathrm{Db}$ & $10,47 \mathrm{Cc}$ \\
\hline & $\mathbf{T 9}$ & $\mathbf{T 1 0}$ & $\mathbf{T 1 1}$ & $\mathbf{T 1 2}$ & $\mathbf{T 1 3}$ & $\mathbf{T 1 4}$ & $\mathbf{T 1 5}$ & $\mathbf{T 1 6}$ \\
\hline $\mathbf{1 5}$ & $31,47 \mathrm{Ac}$ & $35,20 \mathrm{Ab}$ & $34,88 \mathrm{Ab}$ & $34,73 \mathrm{Ab}$ & $36,75 \mathrm{Aa}$ & $32,01 \mathrm{Ac}$ & $34,70 \mathrm{Ab}$ & $33,37 \mathrm{Abc}$ \\
$\mathbf{3 0}$ & $22,20 \mathrm{Bd}$ & $33,10 \mathrm{Aa}$ & $30,03 \mathrm{Ab}$ & $28,30 \mathrm{Ab}$ & $34,43 \mathrm{Aa}$ & $24,04 \mathrm{Bd}$ & $32,03 \mathrm{Aa}$ & $29,05 \mathrm{Ab}$ \\
$\mathbf{4 5}$ & $15,43 \mathrm{Cd}$ & $28,90 \mathrm{Ba}$ & $23,54 \mathrm{Bb}$ & $21,03 \mathrm{Bc}$ & $31,90 \mathrm{Ba}$ & $16,50 \mathrm{Cd}$ & $26,42 \mathrm{Cb}$ & $20,33 \mathrm{Bc}$ \\
$\mathbf{6 0}$ & $9,03 \mathrm{Dd}$ & $22,36 \mathrm{Cb}$ & $17,18 \mathrm{Cc}$ & $15,13 \mathrm{Cc}$ & $28,71 \mathrm{Ba}$ & $10,07 \mathrm{Dd}$ & $20,29 \mathrm{Db}$ & $14,52 \mathrm{Cc}$ \\
$\mathbf{7 5}$ & $5,02 \mathrm{Dd}$ & $16,90 \mathrm{Db}$ & $10,66 \mathrm{Dc}$ & $8,60 \mathrm{Dc}$ & $26,30 \mathrm{Ca}$ & $5,77 \mathrm{Dd}$ & $16,40 \mathrm{Db}$ & $9,29 \mathrm{Cc}$
\end{tabular}

As médias seguidas das mesmas letras minúscula (linha) e maiúscula (coluna) não diferem entre si, ao nível de 5\% de probabilidade, pelo teste de Tukey.

TABELA 3 - Teor de clorofila, expresso em mg.10 $\mathrm{cm}^{-2}$, em limas-ácidas produzidas no Estado de Roraima, frigoarmazenadas por 75 dias a $1 \pm 0,5^{\circ} \mathrm{C}$ e $92 \pm 3 \%$ de U.R., Boa Vista-RR -2006 .

\begin{tabular}{c|cccccccc}
\hline & $\mathbf{T 1}$ & $\mathbf{T 2}$ & $\mathbf{T 3}$ & $\mathbf{T 4}$ & $\mathbf{T 5}$ & $\mathbf{T 6}$ & T7 & T8 \\
\hline $\mathbf{1 5}$ & $16,44 \mathrm{Ab}$ & $20,03 \mathrm{Aa}$ & $17,03 \mathrm{Ab}$ & $16,84 \mathrm{Ab}$ & $22,13 \mathrm{Aa}$ & $16,04 \mathrm{Ab}$ & $20,20 \mathrm{Aa}$ & $17,71 \mathrm{Ab}$ \\
$\mathbf{3 0}$ & $13,20 \mathrm{Bb}$ & $18,44 \mathrm{Aa}$ & $14,23 \mathrm{Ab}$ & $14,05 \mathrm{Ab}$ & $21,20 \mathrm{Aa}$ & $13,40 \mathrm{Ab}$ & $17,45 \mathrm{Aa}$ & $14,11 \mathrm{Bb}$ \\
$\mathbf{4 5}$ & $11,30 \mathrm{Cc}$ & $16,91 \mathrm{Ab}$ & $11,41 \mathrm{Bc}$ & $10,45 \mathrm{Bc}$ & $19,93 \mathrm{Ba}$ & $10,80 \mathrm{Bc}$ & $15,54 \mathrm{Bb}$ & $11,05 \mathrm{Cc}$ \\
$\mathbf{6 0}$ & $8,68 \mathrm{Cc}$ & $13,90 \mathrm{Bb}$ & $8,45 \mathrm{Bc}$ & $8,08 \mathrm{Bc}$ & $18,33 \mathrm{Ba}$ & $8,33 \mathrm{Cc}$ & $12,90 \mathrm{Cb}$ & $9,12 \mathrm{Cc}$ \\
$\mathbf{7 5}$ & $6,01 \mathrm{Dc}$ & $10,80 \mathrm{Cb}$ & $5,31 \mathrm{Cc}$ & $4,80 \mathrm{Cc}$ & $17,32 \mathrm{Ba}$ & $5,02 \mathrm{Dc}$ & $7,80 \mathrm{Dbc}$ & $4,55 \mathrm{Dc}$ \\
\hline & $\mathbf{T 9}$ & $\mathbf{T 1 0}$ & $\mathbf{T 1 1}$ & $\mathbf{T 1 2}$ & $\mathbf{T 1 3}$ & $\mathbf{T 1 4}$ & $\mathbf{T 1 5}$ & $\mathbf{T 1 6}$ \\
\hline $\mathbf{1 5}$ & $17,34 \mathrm{Ab}$ & $19,40 \mathrm{Aa}$ & $18,12 \mathrm{Ab}$ & $16,65 \mathrm{Ab}$ & $22,90 \mathrm{Aa}$ & $16,77 \mathrm{Ab}$ & $19,55 \mathrm{Aa}$ & $18,00 \mathrm{Ab}$ \\
$\mathbf{3 0}$ & $12,78 \mathrm{Bb}$ & $17,12 \mathrm{Aa}$ & $13,32 \mathrm{Ab}$ & $13,10 \mathrm{Ab}$ & $20,31 \mathrm{Aa}$ & $12,49 \mathrm{Bb}$ & $16,30 \mathrm{Ba}$ & $13,30 \mathrm{Bb}$ \\
$\mathbf{4 5}$ & $9,90 \mathrm{Cc}$ & $14,32 \mathrm{Bb}$ & $9,60 \mathrm{Bc}$ & $9,23 \mathrm{Bc}$ & $19,10 \mathrm{Ba}$ & $9,02 \mathrm{Cc}$ & $15,02 \mathrm{Bb}$ & $10,10 \mathrm{Cc}$ \\
$\mathbf{6 0}$ & $7,54 \mathrm{Dc}$ & $12,20 \mathrm{Bb}$ & $8,02 \mathrm{Bc}$ & $8,12 \mathrm{Bc}$ & $17,42 \mathrm{Ba}$ & $7,90 \mathrm{Cc}$ & $11,80 \mathrm{Cb}$ & $8,84 \mathrm{Cc}$ \\
$\mathbf{7 5}$ & $5,64 \mathrm{Dc}$ & $10,85 \mathrm{Cb}$ & $5,43 \mathrm{Cc}$ & $4,99 \mathrm{Cc}$ & $16,99 \mathrm{Ba}$ & $5,23 \mathrm{Dc}$ & $7,44 \mathrm{Dbc}$ & $4,45 \mathrm{Dc}$ \\
\hline
\end{tabular}

As médias seguidas das mesmas letras minúscula (linha) e maiúscula (coluna) não diferem entre si, ao nível de 5\% de probabilidade, pelo teste de Tukey.

TABELA 4 - Perda de massa fresca, expressa em porcentagens médias, em limas-ácidas produzidas no Estado de Roraima, frigoarmazenadas por 75 dias a $1 \pm 0,5^{\circ} \mathrm{C}$ e $92 \pm 3 \%$ de U.R., Boa Vista-RR -2006 .

\begin{tabular}{c|cccccccc}
\hline & $\mathbf{T 1}$ & $\mathbf{T 2}$ & $\mathbf{T 3}$ & $\mathbf{T 4}$ & $\mathbf{T 5}$ & $\mathbf{T 6}$ & $\mathbf{T} 7$ & T8 \\
\hline $\mathbf{1 5}$ & $4,12 \mathrm{Dab}$ & $3,79 \mathrm{Cb}$ & $4,22 \mathrm{Dab}$ & $5,03 \mathrm{Da}$ & $1,90 \mathrm{Bc}$ & $4,71 \mathrm{Cab}$ & $3,96 \mathrm{Cb}$ & $5,23 \mathrm{Ca}$ \\
$\mathbf{3 0}$ & $7,70 \mathrm{Ca}$ & $5,77 \mathrm{Ca}$ & $7,50 \mathrm{Ca}$ & $8,07 \mathrm{Ca}$ & $2,75 \mathrm{Cb}$ & $6,44 \mathrm{Ca}$ & $5,73 \mathrm{Ca}$ & $7,21 \mathrm{Ca}$ \\
$\mathbf{4 5}$ & $9,83 \mathrm{Ca}$ & $8,41 \mathrm{Ba}$ & $9,91 \mathrm{Ca}$ & $9,37 \mathrm{Ca}$ & $3,52 \mathrm{Bb}$ & $10,21 \mathrm{Ba}$ & $9,02 \mathrm{Ba}$ & $9,14 \mathrm{Ba}$ \\
$\mathbf{6 0}$ & $13,75 \mathrm{Ba}$ & $9,23 \mathrm{Ab}$ & $13,81 \mathrm{Ba}$ & $13,98 \mathrm{Ba}$ & $4,88 \mathrm{Ac}$ & $13,85 \mathrm{Aa}$ & $11,03 \mathrm{Aab}$ & $12,12 \mathrm{Ba}$ \\
$\mathbf{7 5}$ & $18,30 \mathrm{Aa}$ & $12,34 \mathrm{Ab}$ & $18,44 \mathrm{Aa}$ & $19,12 \mathrm{Aa}$ & $5,04 \mathrm{Ac}$ & $17,88 \mathrm{Aa}$ & $13,01 \mathrm{Ab}$ & $16,99 \mathrm{Aa}$ \\
\hline & $\mathbf{T 9}$ & $\mathbf{T 1 0}$ & $\mathbf{T 1 1}$ & $\mathbf{T 1 2}$ & $\mathbf{T 1 3}$ & $\mathbf{T 1 4}$ & $\mathbf{T 1 5}$ & $\mathbf{T 1 6}$ \\
\hline $\mathbf{1 5}$ & $5,51 \mathrm{Ea}$ & $3,87 \mathrm{Cb}$ & $4,82 \mathrm{Da}$ & $4,92 \mathrm{Da}$ & $2,02 \mathrm{Cc}$ & $3,47 \mathrm{Cb}$ & $3,65 \mathrm{Cb}$ & $4,57 \mathrm{Dab}$ \\
$\mathbf{3 0}$ & $7,20 \mathrm{Da}$ & $5,62 \mathrm{Ca}$ & $7,88 \mathrm{Ca}$ & $7,42 \mathrm{Ca}$ & $2,55 \mathrm{Cb}$ & $6,68 \mathrm{Ca}$ & $6,00 \mathrm{Ca}$ & $7,91 \mathrm{Ca}$ \\
$\mathbf{4 5}$ & $10,33 \mathrm{Ca}$ & $8,22 \mathrm{Ba}$ & $10,02 \mathrm{Ba}$ & $10,44 \mathrm{Ca}$ & $3,98 \mathrm{Bb}$ & $9,10 \mathrm{Ba}$ & $8,61 \mathrm{Ba}$ & $10,04 \mathrm{Ba}$ \\
$\mathbf{6 0}$ & $15,32 \mathrm{Ba}$ & $9,91 \mathrm{Ab}$ & $14,73 \mathrm{Aa}$ & $16,05 \mathrm{Ba}$ & $4,95 \mathrm{Ac}$ & $13,92 \mathrm{Aa}$ & $10,87 \mathrm{Aab}$ & $14,18 \mathrm{Ba}$ \\
$\mathbf{7 5}$ & $19,98 \mathrm{Aa}$ & $12,99 \mathrm{Ab}$ & $18,34 \mathrm{Aa}$ & $20,10 \mathrm{Aa}$ & $5,32 \mathrm{Ac}$ & $17,90 \mathrm{Aa}$ & $13,14 \mathrm{Ab}$ & $20,11 \mathrm{Aa}$ \\
\hline
\end{tabular}

As médias seguidas das mesmas letras minúscula (linha) e maiúscula (coluna) não diferem entre si, ao nível de 5\% de probabilidade, pelo teste de Tukey 
TABELA 5 - Danos de frio, expresso através de escala subjetiva de valores, em limas-ácidas Tahiti produzidas no Estado de Roraima, frigoarmazenadas por 75 dias a $1 \pm 0,5^{\circ} \mathrm{C}$ e $92 \pm 3 \%$ de U.R., Boa Vista-RR -2006.

\begin{tabular}{l|cccccccc}
\hline & T1 & T2 & T3 & T4 & T5 & T6 & T7 & T8 \\
\hline $\mathbf{1 5}$ & $1,2 \mathrm{Ba}$ & $0 \mathrm{Ab}$ & $0 \mathrm{Ab}$ & $0 \mathrm{Ab}$ & $0 \mathrm{Ab}$ & $1,3 \mathrm{Ba}$ & $0 \mathrm{Ab}$ & $0 \mathrm{Ab}$ \\
$\mathbf{3 0}$ & $1,8 \mathrm{Ba}$ & $0 \mathrm{Ab}$ & $0 \mathrm{Ab}$ & $0 \mathrm{Ab}$ & $0 \mathrm{Ab}$ & $2,4 \mathrm{Ba}$ & $0 \mathrm{Ab}$ & $0 \mathrm{Ab}$ \\
$\mathbf{4 5}$ & $2,4 \mathrm{Ba}$ & $0 \mathrm{Ab}$ & $0 \mathrm{Ab}$ & $0 \mathrm{Ab}$ & $0 \mathrm{Ab}$ & $3 \mathrm{Aa}$ & $0 \mathrm{Ab}$ & $0 \mathrm{Ab}$ \\
$\mathbf{6 0}$ & $2,9 \mathrm{Aa}$ & $0 \mathrm{Ab}$ & $0 \mathrm{Ab}$ & $0 \mathrm{Ab}$ & $0 \mathrm{Ab}$ & $3,2 \mathrm{Aa}$ & $0 \mathrm{Ab}$ & $0 \mathrm{Ab}$ \\
$\mathbf{7 5}$ & $3,2 \mathrm{Aa}$ & $0 \mathrm{Ab}$ & $0 \mathrm{Ab}$ & $0 \mathrm{Ab}$ & $0 \mathrm{Ab}$ & $4 \mathrm{Aa}$ & $0 \mathrm{Ab}$ & $0 \mathrm{Ab}$ \\
\hline $\mathbf{1 5}$ & $\mathbf{T 9}$ & $\mathbf{T 1 0}$ & $\mathbf{T 1 1}$ & $\mathbf{T 1 2}$ & $\mathbf{T 1 3}$ & $\mathbf{T 1 4}$ & $\mathbf{T 1 5}$ & $0 \mathrm{T16}$ \\
$\mathbf{3 0}$ & $1,5 \mathrm{Ba}$ & $0 \mathrm{Ab}$ & $0 \mathrm{Ab}$ & $0 \mathrm{Ab}$ & $0 \mathrm{Ab}$ & $2,5 \mathrm{Ba}$ & $0 \mathrm{Ab}$ & $0 \mathrm{Ab}$ \\
$\mathbf{4 5}$ & $2,5 \mathrm{Ba}$ & $0 \mathrm{Ab}$ & $0 \mathrm{Ab}$ & $0 \mathrm{Ab}$ & $0 \mathrm{Ab}$ & $3,2 \mathrm{Aa}$ & $0 \mathrm{Ab}$ & $0 \mathrm{Ab}$ \\
$\mathbf{6 0}$ & $3,2 \mathrm{Aa}$ & $0 \mathrm{Ab}$ & $0 \mathrm{Ab}$ & $0 \mathrm{Ab}$ & $0 \mathrm{Ab}$ & $4 \mathrm{Aa}$ & $0 \mathrm{Ab}$ & $0 \mathrm{Ab}$ \\
$\mathbf{7 5}$ & $4 \mathrm{Aa}$ & $0 \mathrm{Ab}$ & $0 \mathrm{Ab}$ & $0 \mathrm{Ab}$ & $0 \mathrm{Ab}$ & $4 \mathrm{Aa}$ & $0 \mathrm{Ab}$ & $0 \mathrm{Ab}$ \\
\hline
\end{tabular}

As médias seguidas das mesmas letras minúscula (linha) e maiúscula (coluna) não diferem entre si, ao nível de 5\% de probabilidade, pelo teste de Tukey.

TABELA 6 - Aspecto visual, expresso através da média dos valores obtidos em 3 escalas não-paramétricas (rugosidade, murchamento e podridões na casca dos frutos), em limas-ácidas Tahiti produzidas no Estado de Roraima, frigoarmazenadas por 75 dias a $1 \pm 0,5^{\circ} \mathrm{C} \mathrm{e} 92 \pm 3 \%$ de U.R., Boa Vista-RR -2006 .

\begin{tabular}{l|cccccccc}
\hline & $\mathbf{T 1}$ & $\mathbf{T 2}$ & $\mathbf{T 3}$ & $\mathbf{T 4}$ & $\mathbf{T 5}$ & $\mathbf{T 6}$ & T7 & T8 \\
\hline $\mathbf{1 5}$ & $7,8 \mathrm{Ab}$ & $9,2 \mathrm{Aa}$ & $9,3 \mathrm{Aa}$ & $7,8 \mathrm{Ab}$ & $9,6 \mathrm{Aa}$ & $7,6 \mathrm{Ab}$ & $9,3 \mathrm{Aa}$ & $9,2 \mathrm{Aa}$ \\
$\mathbf{3 0}$ & $7,3 \mathrm{Ab}$ & $8,1 \mathrm{Aab}$ & $7,7 \mathrm{Ab}$ & $6,4 \mathrm{Bc}$ & $9 \mathrm{Aa}$ & $6,4 \mathrm{Bc}$ & $7,9 \mathrm{Bab}$ & $7,5 \mathrm{Bb}$ \\
$\mathbf{4 5}$ & $6,5 \mathrm{Bb}$ & $7,3 \mathrm{Ab}$ & $6 \mathrm{Bb}$ & $5,1 \mathrm{Cc}$ & $8,7 \mathrm{Aa}$ & $4,3 \mathrm{Cc}$ & $6,4 \mathrm{BCb}$ & $6,7 \mathrm{Bb}$ \\
$\mathbf{6 0}$ & $4,8 \mathrm{Cc}$ & $6,1 \mathrm{Bb}$ & $5,5 \mathrm{Bbc}$ & $4,2 \mathrm{Cc}$ & $7,8 \mathrm{Ba}$ & $3 \mathrm{Dd}$ & $5,4 \mathrm{Cbc}$ & $5,4 \mathrm{BCbc}$ \\
$\mathbf{7 5}$ & $3,4 \mathrm{Cc}$ & $3,5 \mathrm{Cc}$ & $3,5 \mathrm{Cc}$ & $2,5 \mathrm{CDd}$ & $7,2 \mathrm{Ba}$ & $2 \mathrm{Dd}$ & $5,1 \mathrm{Cb}$ & $3,5 \mathrm{Cc}$ \\
\hline & $\mathbf{T 9}$ & $\mathbf{T 1 0}$ & $\mathbf{T 1 1}$ & $\mathbf{T 1 2}$ & $\mathbf{T 1 3}$ & $\mathbf{T 1 4}$ & $\mathbf{T 1 5}$ & $\mathbf{T 1 6}$ \\
\hline $\mathbf{1 5}$ & $7,2 \mathrm{Ab}$ & $9,5 \mathrm{Aa}$ & $9,4 \mathrm{Aa}$ & $7 \mathrm{Ab}$ & $9,5 \mathrm{Aa}$ & $8 \mathrm{Ab}$ & $9 \mathrm{Aa}$ & $9,3 \mathrm{Aa}$ \\
$\mathbf{3 0}$ & $6,4 \mathrm{Bc}$ & $7,4 \mathrm{Ab}$ & $6,8 \mathrm{Bc}$ & $5,5 \mathrm{BCd}$ & $9,2 \mathrm{Aa}$ & $6,5 \mathrm{Bc}$ & $7,8 \mathrm{Bab}$ & $7,6 \mathrm{Bb}$ \\
$\mathbf{4 5}$ & $6,2 \mathrm{Bb}$ & $6,6 \mathrm{Bb}$ & $5,2 \mathrm{Bc}$ & $4,8 \mathrm{Cc}$ & $9 \mathrm{Aa}$ & $4,5 \mathrm{Cc}$ & $7 \mathrm{BCb}$ & $6,4 \mathrm{Bb}$ \\
$\mathbf{6 0}$ & $3,5 \mathrm{Cd}$ & $5,4 \mathrm{Bbc}$ & $3,9 \mathrm{Ccd}$ & $2,7 \mathrm{CDd}$ & $8,3 \mathrm{Ba}$ & $3,3 \mathrm{CDd}$ & $5,5 \mathrm{Cc}$ & $5,3 \mathrm{BCbc}$ \\
$\mathbf{7 5}$ & $1,8 \mathrm{Dd}$ & $3,5 \mathrm{Cc}$ & $1,9 \mathrm{Dd}$ & $1,9 \mathrm{Dd}$ & $7,3 \mathrm{Ba}$ & $2 \mathrm{Dd}$ & $4,8 \mathrm{Cb}$ & $3,4 \mathrm{Cc}$ \\
\hline
\end{tabular}

As médias seguidas das mesmas letras minúscula (linha) e maiúscula (coluna) não diferem entre si, ao nível de 5\% de probabilidade, pelo teste de Tukey.

\section{CONCLUSÃO}

O aquecimento intermitente a $20^{\circ} \mathrm{C}$, aplicado aos 5 e 10 dias em limas-ácidas Tahiti, durante o armazenamento refrigerado a $1 \pm 0,5{ }^{\circ} \mathrm{C}$ e $92 \pm 5 \%$ de UR., permite o controle satisfatório dos danos de frio, bem como a manutenção dos atributos químicos e físicos dos frutos, durante 75 dias nessas condições.

\section{REFERÊNCIAS}

AGRIANUAL 2005: anuário da agricultura brasileira. São Paulo: FNP Consultoria e Comércio, 2004. p.306-311.

AGRIANUAL 2006: anuário estatístico da agricultura brasileira. São Paulo: FNP Consultoria e Comércio, 2005. 506 p.

CHITARRA, M.I.F.; CHITARRA, A.B. Pós-Colheita de frutas e hortaliças fisiologia e manejo. 2. ed. rev. ampl. Lavras: UFLA, 2005. $785 \mathrm{p}$.

COELHO, Y., LORDÊLO, C.; CALDAS, R. Cuidados com a lima ácida 'Tahiti' da Bahia para valorizar o comércio com a Europa. 
Disponível: <http://www.nordesterural.com.br/>. Acesso em: 06 abr 2007.

EMBRAPA. Sistema de produção para pequenos produtores de citros do nordeste. Campinas, SP: Embrapa Informática Agropecuária. Disponível em: < http://www.cnptia.embrapa.br/ >. Acesso em: 03 mar. 2007.

GOLDSCHMIDT, E.E. Ripening of citrus and other non-climateric fruits: a role for ethylene. Acta Horticulturae, Leuven, n.463, p.325-334, 1997.

JACOMINO, A.P.; MENDONCA, K.; KLUGE, R.A. Armazenamento refrigerado de limões 'Siciliano' tratados com etileno. Revista Brasileira de Fruticultura, Jaboticabal, v.25, n. 1, p. $45-48,2003$.

JOMORI, M.L.L Resistência de lima-ácida Tahiti à baixa temperatura: tratamentos térmicos e envolvimento do etileno. 2005. 116 f. Dissertação (Mestrado) - Escola Superior de Agricultura "Luiz de Queiroz", Universidade Estadual Paulista, Piracicaba. 2005

JOMORI, M.L.L.; KLUGE, R. A.; JACOMINO, A.P. Cold storage of 'Tahiti' lime treated with 1-methylcyclopropene. Scientia Agricola, Piracicaba, v. 60, n. 4, p. 785-788, 2003a.

JOMORI, M.L.L.; KLUGE, R. A.; JACOMINO, A.P; TAVARES, S. Conservação refrigerada de lima ácida 'Tahiti': uso de 1metilciclopropeno, ácido giberélico e cera. Revista Brasileira de Fruticultura, Jaboticabal, v. 25, n. 3, p. 406-409, 2003 b.

KLUGE, R. A.; JOMORI, M.L.L.; JACOMINO, A.P.; VITTI, M.C.D.; PADULA, M. Intermittent warming in 'Tahiti' lime treated with an ethylene inhibitor. Postharvest Biology And Technology, Wageningen, v. 29, n. 2, p. 195-203, 2003a.

KLUGE, R.A.; AZEVEDO, R.A. de; JOMORI, M.L.L.; EDAGI, F.K.; JACOMINO, A.P.; GAZIOLA, S.A.; AGUILA, J.S.D. Efeitos de tratamentos térmicos aplicados sobre frutas cítricas armazenadas sob refrigeração. Ciência Rural, Santa Maria, v. 36, n. 5, p. 1388-1396, 2006.

KLUGE, R.A.; JOMORI, M.L.L; JACOMINO, A.P.; VITTI, M.C.D. Intermittent warming in 'Tahiti' lime to prevent chilling injury in cold storage. Scientia Agricola, Piracicaba, v. 60, n. 4, p. 729-734, $2003 b$.

LEE, S.K.; YOUNG, R.E. Temperature sensitivity of avocado fruit in relation to $\mathrm{C}_{2} \mathrm{H}_{4}$ treatment. Journal of the American Society for Horticultural Science, Alexandria, v.5, n.109, p. 689-692. 1984

MANFROI, V.; LUCCHESE, O.A.; CARVALHO, R.I.N.; FIOVARANÇO, J.C.; BENDER, R.J. Efeito do 2,4-D na frigoconservação de limão Tahiti (Citrus latifolia Tanaka). Boletim do CEPPA, Curitiba, v. 14, n. 1, p. 77-88, 1996.

PESIS, E.; ACKERMAN, M.; BEN-ARIE, R.; FEYGENBERG, O.; FENG, X.; APELBAUM, A.; GOREN, R. \& PRUSKY, D.. Ethylene involvement in chilling injury symptoms of avocado during cold storage. Postharvest Biology and Technology, Amsterdan, n.24, p.171-181,2002

PORAT, R.; WEISS, B.; COHEN, L.; DAUS, A.; GOREN, R.; DROBY, S. Effects of ethylene and 1-methylcyclopropene on the postharvest qualities of "Shamouti" oranges. Postharvest Biology and Technology, Amsterdan, v.15, n.2, p.155-163, 1999.

RODOV, V.; AGAR, T.; PERETZ, J.; NAFUSSI, B.; KIM, J.J.; BENYEHOSHUA, S. Effect of combined application of heat treatments and plastic packaging on keeping quality of "Oroblanco" fruit (Citrus grandis L. x C. paradisi Macf.). Postharvest Biology and Technology, Amsterdan, v.20, p.287-294, 2000.

SALA, J.M.; LAFUENTE, M.T. Catalase in the heat-induced chilling tolerance of cold-stored hybrid Fortune mandarin fruits. Journal of Agriculture and Food Chemistry, Washington, v.47, p.2410-2414, 1999.

SALVADOR, A.; ARNAL, L.; MONTERDE, A.; CUQUERELLA, $\mathrm{J}$. Reduction of chilling injury symptoms in persimmon fruit cv. 'Rojo Brillante' by 1-MCP. Postharvest Biology and Technology, Amsterdam, v.33, p.285-291, 2004.

SCHIRRA, M., MULAS, M., FADDA, A., MIGNANA, I.; LURIE, S. Chemical and quality traits of 'Olinda' and 'Campbell' oranges after heat treatment at 44 or $46^{\circ} \mathrm{C}$ for fruit fly disinfestations. Lebensmittel Wissenschaft und Technologie, Zurich, n.38, p.519522.2005.

SCHIRRA, M.; COHEN, E. Long-term storage of "Olinda" oranges under chilling and intermittent warming temperatures. Postharvest Biology and Technology, Amsterdan, v.16, p.63-69, 1999.

SELVARAJAH, S.; BAUCHOT, A.D.; JOHN, P. Internal browning in cold-stored pineapples is suppressed by a postharvest application of 1-methylcyclopropene. Postharvest Biology and Technology, Amsterdam, v.23, n.2, p.167-170, 2001.

VANDERZANT, C.; SPLITTSTOESSER, D.F. Compendium of methods for the microbiological examination of foods. Washington: American Public Health Association, 1992. 600p.

YUEN, C.M.C.; TRIDJAJA N.O.; WILLS R.B.H.; WILD B. L. Chilling injury development of 'Tahitian' lime, 'Emperor' mandarin, 'Marsh' grapefruit and 'Valencia' orange.

Journal of the Science of Food and Agriculture, London, v.67, n.3, p.335-339, 1995. 